\title{
LA PAURA. RIFLESSIONI INTERDISCIPLINARI PER UN DIBATTITO CONTEMPORANEO SU VIOLENZA, ORDINE, SICUREZZA
}

\author{
CLAUDIA STORTI (*) e FLORIANA COLAO (**)
}

Nota presentata dal s.c. Claudia Storti

(Adunanza del 3 dicembre 2020)

SUNTO. - Il libro La paura. Riflessioni interdisciplinari per un dibattito contemporaneo su violenza, ordine, sicurezza offre numerose prospettive di riflessione interdisciplinare su un aspetto della vita delle società che ha caratteri di continuità tra passato e presente e forte influenza sulla configurazione dei sistemi penali. Se, come istinto, la paura costituisce uno strumento naturale di difesa per l'individuo, la paura 'collettiva' è fenomeno che, nella convivenza sociale, si presta a numerose declinazioni e non tutte di segno positivo, come si vede, ad esempio, nell'attuale ipertrofia del diritto penale. La paura è talvolta scatenata da cause oggettive (da fenomeni naturali come le catastrofi, le epidemie, le carestie, oppure da guerre e rivoluzioni); in altri casi, invece, è suscitata dalle cosiddette 'culture della paura', tese a ingenerare o a accrescere con la propaganda e la retorica o con atti di violenza (basti pensare alla 'funzione' degli atti di terrorismo) insicurezza di fronte a 'nemici' talvolta veri, talvolta 'presunti' allo scopo di suscitare ricerca di protezione e sicurezza e con questo di rafforzare il potere di chi offre tutela anche con gli strumenti del diritto penale.

ABSTRACT. - The book Riflessioni interdisciplinari per un dibattito contemporaneo su violenza, ordine, sicurezza offers an interesting interdisciplinary perspective on aspects of the social life that undoubtedly show continuity between past and present and that

(*) Professore ordinario di Storia del diritto medievale e moderno, Università degli Studi di Milano, Dipartimento di Diritto pubblico italiano e sovranazionale, Milano, Italy. E-mail: claudia.storti@unimi.it

(j*) Professore ordinario di Storia del diritto medievale e moderno, Università di Siena, Dipartimento di Giurisprudenza, Siena, Italy. E-mail: floriana.colao@unisi.it 
exert a strong influence on criminal systems. If fear, as an instinct, constitutes a natural defense tool for the individual, 'collective' fear is a phenomenon open to different declinations, not all positive, as we will see, for example, in the current hypertrophy of the criminal law. Sometimes collective fear is triggered by objective causes (e.g. by natural catastrophes, epidemics, famines, wars or revolutions); sometimes, instead, collective fear is generated by the so called 'culture of fear'. Such culture instills and amplifies a sense of insecurity towards true or presumed enemies by using propaganda, rhetoric or violence (just think at the 'function' of terrorism). This insecurity, in turn, drives people to search safety and strengthens the power of those who offer protection even with the tools of the criminal law.

\section{Claudia Storti}

Ringrazio il Presidente e il Consiglio dell'Istituto Lombardo per aver accolto la proposta di una nota sul libro La paura. Riflessioni interdisciplinari per un dibattito contemporaneo su violenza, ordine, sicurez$z a^{1}$. L'avevo sottoposta alla loro attenzione prima ancora che si potessero seppur intravedere i primi segni della paura totale e globale dei nostri giorni, quella della pandemia, che, come si potrebbe dire, sembra aver un po' offuscato e fatto perdere spessore, almeno nell'opinione pubblica, a molte altre paure.

L'idea di presentare un libro sulla paura incentrato sulla questione penale, come 'parola chiave' del dibattito attuale su violenza ordine e sicurezza era stata ispirata sia dall'attualità del tema, sia dalle caratteristiche del progetto di ricerca e dalla particolarità dell'itinerario che ha portato alla sua scrittura.

Il libro sulla paura nasce nell'ambito di un progetto più ampio: quello di un laboratorio di riflessione interdisciplinare su alcuni concetti e temi cardine per l'indagine sulla complessità del problema penale (paura, dubbio, castigo) con precipuo riguardo alla paura della crimi-

1 Quaderno di Storia del penale e della giustizia, 1(2019) La paura. Riflessioni interdisciplinari per un dibattito contemporaneo su violenza, ordine, sicurezza, http://eum.unimc.it/it/catalogo/646-quaderno-di-storia-del-penale-e-della-giustizia-12019-la-paura (d'ora innanzi = La paura). Nel frattempo è già uscita la continuazione di questa rivista: Quaderno di storia del penale e della giustizia 2(2020) Il Dubbio. Riflessioni interdisciplinari per un dibattito contemporaneo su certezza, giustizia, mass media e diritto di punire bttp://eum.unimc.it/it/catalogo/696-quaderno-di-storia-delpenale-e-della-giustizia-2-2020-il-dubbio 
nalità e quindi nell'ambito del tema della sicurezza o, meglio, della crescente in-sicurezza sociale, ormai oggetto di numerosi studi². Nel 2018, il laboratorio, come sottolineato nella nota introduttiva al volume, ha coinvolto non solo storici e storici del diritto ma anche studiosi di antropologia, sociologia, filosofia, psicologia e politologia con lo scopo di affrontare in una «visione plurale dinamica e integrata» i caratteri del «sistema penale» e della «giustizia penale» dei tempi odierni.

Tale laboratorio è stato ideato dagli allievi (Luigi Lacché e Massimo Meccarelli) del grandissimo storico del diritto penale Mario Sbriccoli, scomparso prematuramente nel 2005, che ha aperto nuovi percorsi per l'indagine e la comprensione del fenomeno penale dal medioevo alla contemporaneità lasciando un'eredità indelebile ${ }^{4}$. La stessa immagine raffigurata sulla copertina della paura è tratta da un disegno di Pablo Picasso, che Mario Sbriccoli aveva riprodotto nei suoi ex libris (Fig. 1).

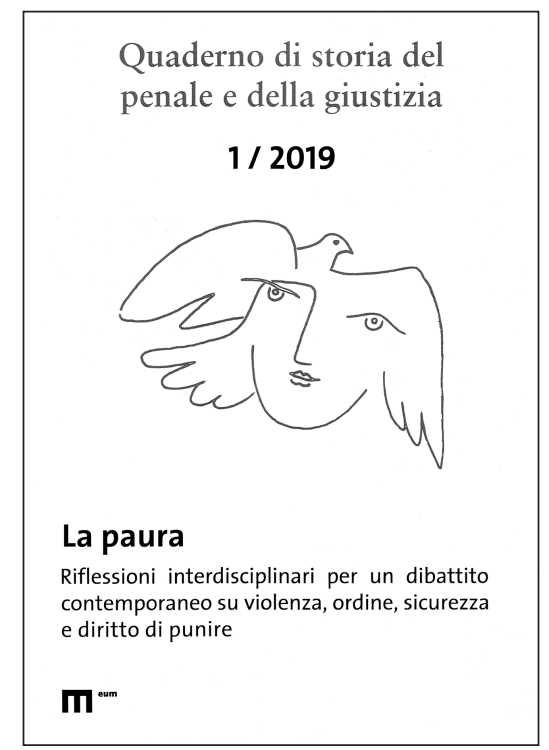

Fig. 1.

2 Per i riferimenti cfr. R. Cornelli, La paura nel campo penale: una storia del presente, La paura, 63-90, in part. 63.

3 L. Lacchè, M. Meccarelli, Paura in criminalibus: il problema penale tra sentimento umano e dinamiche sociali, La paura, 7-13, in part. 9.

4 Gli straordinari risultati della ricerca di Mario Sbriccoli sono stati rilevati da 
Per uno dei frequenti repentini inconvenienti di questi tempi strani, Luigi Lacchè, già Rettore dell'Università di Macerata e storico del diritto costituzionale e del diritto penale, che avrebbe dovuto illustrarlo oggi non può essere presente 5 . Floriana Colao, professore ordinario di storia del diritto nell'Università di Siena, ha accettato di discuterne con noi non solo con la sua consueta disponibilità e entusiasmo per la ricerca, ma anche per il suo spirito di condivisione e continuo desiderio di dialogo e di contraddittorio sui risultati degli studi. Studi che accomunano da tanti anni, come è attestato dalle ricerche congiunte, alcuni storici del diritto delle Università di Macerata, di Siena, di Milano con il coinvolgimento di colleghi di altre università italiane, tra le quali mi limito a citare quelle su Processo penale e opinione pubblica ${ }^{6}$, sulla, cosiddetta, Legislazione grigia ${ }^{7}$, su Giustizia penale e politica in Italia tra Otto e Novecento ${ }^{8}$, sul Diritto del Duce e, naturalmente, appunto sulla Paura.

Floriana Colao, studiosa di tantissimi ambiti della storia giuridica, e autrice di studi e monografie fino all'età contemporanea, si è dedicata negli ultimi anni soprattutto allo studio del diritto e del processo pena-

numerosissimi colleghi in Penale, giustizia, potere. Metodi, ricerche, storiografie per ricordare Mario Sbriccoli, a cura di L. Lacchè, Macerata, eum, 2007 e in Principio di legalità e diritto penale, Milano, Giuffrè, 2007 (Quaderni fiorentini, 36 2007). Tra le sue pubblicazioni più importanti M. Sbriccoli, L'interpretazione dello Statuto: contributo allo studio della funzione dei giuristi nell' età comunale. Milano, A. Giuffrè, 1969; Id., Crimen laesae maiestatis: il problema del reato politico alle soglie della scienza penalistica moderna, Milano: Giuffre, 1974; Id., Ordo iuris: storia e forme dell' esperienza giuridica, Milano, Giuffrè, 2003; Id., Storia del diritto penale e della giustizia: scritti editi e inediti (1972-2007). Milano, Giuffrè, 2009.

5 L. Lacchè, La giustizia per i galantuomini. Ordine e libertà nell'Italia liberale: il dibattito sul carcere preventivo: 1865-1913. Milano: A. Giuffrè, 1990; Id., Non giudicate: antropologia della giustizia e figure dell'opinione pubblica tra Otto e Novecento, Napoli, Satura, 2009; Id., La libertà che guida il popolo: Le tre gloriose giomate del luglio 1830 e le Chartes nel costituzionalismo francese. Bologna, Il Mulino, 2002; Id., History E constitution. Developments in European constitutionalism: the comparative experience of Italy, France, Switzerland and Belgium (19th - 20th centuries), Frankfurt am Main, Vittorio Klostermann, 2016.

6 Processo penale e opinione pubblica in Italia tra Otto e Novecento, a cura di F. Colao, L. Lacchè, C. Storti, Bologna, Il Mulino, 2008.

7 Perpetue appendici e codicilli alle leggi italiane, a cura di F. Colao, L. Lacchè, C. Storti, C. Valsecchi, Macerata, eum, 2011.

8 Giustizia penale e politica in Italia tra Otto e Novecento. Modelli e esperienze tra integrazione e conflitto, a cura di F. Colao, L. Lacchè, C. Storti, Milano, Giuffrè, 2015

9 Il diritto del Duce. Giustizia e repressione nell'Italia fascista, a cura di L. Lacchè, Roma, Donzelli Editore 2015. 
le, e di lei mi limito a ricordare qui soltanto il 'cimento' della pubblicazione della prima monografia sulla storia del diritto e del processo penale nell'età repubblicana, dalla fine del fascismo all'inizio del nuovo secolo e millennio intitolata Giustizia e politica. Il processo penale nell'Italia repubblicana. Il suo coraggio di studiosa alla ricerca di percorsi di interpretazione nell'intreccio tra politica e giustizia è dimostrato dal fatto che la maggior parte del suo testo è dedicata all'indagine del 'problema penale' per un periodo così recente e ancora controverso, quello degli anni Sessanta-Novanta del secolo scorso, dilaniato tra due opposti orientamenti di politica legislativa e giudiziaria. Un periodo, come occorre sottolineare, in parte connotato dal cosiddetto garantismo ossia dal tentativo di attuare i principi costituzionali nel processo penale ancora regolato - seppure con successive modifiche - fino alla fine degli anni Ottanta dal codice di procedura penale fascista, in parte caratterizzato da frequenti provvedimenti di emergenza adottati per far fronte a fenomeni ricorrenti e violenti dello stragismo e del terrorismo, per molti aspetti ancora avvolti nel 'segreto' ${ }^{10}$.

Prima di darle la parola, cercherò di indicare alcune linee sull'impostazione generale del tema della Paura, che si presta a diverse prospettive di lettura a partire proprio dalla coppia sistema penale / paura, facendo un rapido esame degli scritti di carattere generale e introduttivo agli aspetti più tecnici degli effetti della paura nel penale.

Vorrei, però, fare una considerazione preliminare sul fatto che gli storici ritengano di poter dare il loro contributo all' 'analisi dell'attualità' e che, nella loro opinione, lo studio della storia sia utile, se non indispensabile, per orientarsi nei complessi meandri del presente. Non è solo questione di attitudine degli storici, ma anche questione che assilla tutti

10 F. Colao, Giustizia e politica: il processo penale nell'Italia repubblicana. Milano, Giuffrè, 2013. Ricordo, inoltre, tra le sue monografie: F. Colao, Il delitto politico tra Ottocento e Novecento: da delitto fittizio a nemico dello Stato. Milano: A. Giuffrè, 1986; Ead., La giustizia criminale senese nell'età delle riforme leopoldine: post tenebras spero lucem. Milano, Giuffre, 1989; Ead., La libertà di insegnamento e l'autonomia nell'università liberale: norme e progetti per l'istruzione superiore in Italia, 1848 1923, Milano, Giuffrè, 1995; Ead., Progetti di codificazione civile nella Toscana della restaurazione, Bologna, Monduzzi, 1999; Ead., Avvocati del Risorgimento nella Toscana della restaurazione, Bologna, Il mulino, 2006; Ead., Introduzione a L'Italia a 80 anni dalle leggi antiebraiche e a 70 dalla Costituzione. Atti del Convegno tenuto a Siena nei giorni 25 e 26 ottobre 2018, a cura di M. Perini, Pisa, Pacini giuridica, 2019. 
coloro che vogliono riformare l'ordinamento delle facoltà di giurisprudenza, con l'eliminare, appunto, il più possibile gli insegnamenti storico giuridici. Da storico non posso che ribadire l'utilità dell'analisi del presente, prendendo le mosse dalla considerazione che essa è fondamentale, innanzitutto, proprio per la ricerca storica: sono di frequente i caratteri del presente che suggeriscono nuove prospettive di indagine sul passato, le quali aprono, a loro volta, percorsi di analisi altrimenti non avvertibili e consentono, in tal modo, di cogliere aspetti fondamentali sia del passato, sia del presente che sarebbe altrimenti impossibile svelare.

Chiudo la parentesi e vengo all'oggetto del mie considerazioni: di quale paura si tratta e perché è utile mettere a fuoco la paura come chiave di interpretazione dei caratteri del sistema penale odierno?

Da quest'ultimo punto di vista il sistema penale appare connotato da due caratteristiche solo apparentemente in contrasto tra loro. Da un lato, si assiste alla dilatazione del diritto di punire, in una sorta di 'panpunitivismo' o di inflazione di norme che producono una iper-specializzazione dei reati e delle relative pene e una frammentazione della scala penale (pensiamo alla recente categoria dell'omicidio stradale, alla riforma della legittima difesa, ai nuovi reati connessi con l'immigrazione). Dall'altro, il sistema penale - che appare nel complesso più frammentario e, nel contempo, meno efficace - è in crisi: una crisi dovuta non ultimo alla molteplicità dei suoi scopi e dei suoi indirizzi.

In aggiunta, in una crescita continua del fenomeno della giurisdizionalizzazione (iniziata peraltro ormai da parecchi decenni), la giustizia penale, a sua volta, comporta che sempre più di sovente i giudici siano chiamati a dare risposte alla società (ora, come anche nel passato più recente, in campi nei quali il legislatore non riesce o non vuole intervenire) con sentenze che, in molti casi, assumono un valore simbolico e con l'inflizione di pene le quali nell'immaginario comune assumono la funzione della vendetta e dell'esclusione piuttosto che quella della rieducazione (del pensiero illuminista e liberale) per tralasciare poi il discorso sulla funzione 'riparativa' della pena secondo le più innovative tendenze di una parte della cultura e della scienza giuridica ${ }^{11}$.

La sensazione degli ideatori del laboratorio, condivisa dalla maggioranza dei partecipanti, è stata quella di un «circolo vizioso tra [paura

11 G. Mannozzi, Il "ruolo" della paura nel diritto penale e nelle scelte di politica criminale, La paura, 275-294. 
come] allarme sociale, più o meno reale, populismo e rischio di strumentalizzazione politica del diritto penale». Nella riflessione di molti, si tratta di un rischio che riguarda non solo l'Italia, ma molte società democratiche $e^{12}$, in un' età, come la nostra, ormai qualificata come «età dell'incertezza» ${ }^{13}$ per una società dell'astrazione ${ }^{14}$.

Per quanto concerne il secondo termine di questo discorso, ossia la paura nelle sue infinite declinazioni da timore a terrore, non vi è chi non riconosca che si tratta di un sentimento o, meglio, di un istinto non eliminabile né sradicabile ${ }^{15}$, provocato sia da elementi e processi fisici e neurologici individuali, sia da influssi e dinamiche emotivi, di carattere 'collettivo $^{16}$ e di natura culturale e sociale ${ }^{17}$, né che, come la storia dimostra, la paura possa portare a effetti del tutto opposti nella produzione di comportamenti di difesa. Da un lato, la paura può ingenerare una reazione razionale e guidata dal 'discernimento', innanzitutto, sui motivi che l'hanno prodotta; dall'altro, una reazione di annullamento della razionalità.

La storia della società è stata continuamente intessuta di paure, talune persistenti o ricorrenti, altre "nuove"18, a loro volta riconducibili a categorie di fenomeni ora di carattere naturale come la morte, le catastrofi, le malattie/epidemie, le carestie, ora di carattere sociale (guerra, rivoluzioni, complotti, terrorismo), ora legate allo status delle persone: basti pensare a tutti coloro che dal medioevo al XIX secolo sono stati definiti (anche dal punto di vista delle categorie del diritto) come vagabondi, per non dire gli emarginati, gli stranieri e ora, soprattutto, i migranti.

E', inoltre, inutile ricordare che, a fronte della maggioranza di questi fenomeni generatori di paura ora di natura, per così dire, oggettiva, ora determinati, invece, dalle cosiddette 'culture della paura'19, l'antido-

12 L. Lacché, M. Meccarelli, Paura in criminalibus: il problema penale tra sentimento umano e dinamiche sociali, La paura, 7-13, in part. 7.

13 L. Scuccimarra, Semantiche della paura. Un itinerario concettuale, La paura, 17-34, in part. 17.

14 R. Mancini, Dialettiche della paura nella società dell'astrazione, La paura, 35 54 , in part. 35.

15 R. Mancini, Dialettiche della paura cit , 48.

16 R. Cornelli, La paura nel campo penale cit., 65 ss., 71 ss.

17 L. Scuccimarra, Semantiche della paura cit. , 17-20.

18 L. Lacché, M. Meccarelli, Paura in criminalibus cit., 10 e nota 9.

19 L. Scuccimarra, Semantiche della paura cit., 18. 
to' - potremmo dire 'naturale'- è stato ed è identificato nella protezione e nella sicurezza o meglio nella ricerca di protezione e di sicurezza. Tanto che, se si rivolge lo sguardo al passato, proprio intorno a questi due termini paura/ricerca di protezione e sicurezza, secondo autori 'classici', la paura ha rappresentato «l'inizio, la molla della politica ${ }^{20}$.

Il pensiero corre allora inevitabilmente, innanzitutto, alle teorie del contratto sociale come fondamento dello Stato, tra le quali quella del Leviathan di Thomas Hobbes del 1651 costituisce un archetipo di età moderna non solo dell'assolutismo o del dispotismo, ma anche dell'interpretazione e dell'uso della paura. E' sembrato, dunque, a molti corretto assumere Hobbes come paradigma per l'interpretazione di tanti fenomeni odierni nei quali la paura (nel senso di emozione) è utilizzata come strumento ideologico-gerarchico di dominio sulla società, o almeno su una parte della società ${ }^{21}$.

E' indubbio che nella 'secolarizzazione' della paura realizzata da Hobbes, contro il quale, non a caso, prese le distanze Cesare Beccaria ${ }^{22}$, la paura sia strumento funzionale al controllo sociale e veicolo dell'obbedienza, nonché fondamento del diritto di punire. E questo, a sua volta, produce un circolo vizioso e un paradosso. Il rispetto del contratto sociale è originato dalla paura (in natura fear $)^{23}$ che induce alla ricerca di protezione e, con una sorta di paradosso, appunto, produce come risultato finale la paura, o meglio, il terrore della pena (terror of some punishment) nei confronti del 'protettore'. Una variante di tale rappresentazione sarebbe costituita da una conversione dei caratteri della paura che da irrazionale nello stato di natura, si trasformerebbe in razionale quanto si passi al suo utilizzo nello stato "politico ${ }^{24}$, tanto più che lo stesso Hobbes indicava un freno all'assolutismo o al dispotismo nel principio del positivismo legislativo (o di legalità) e in una sorta di auto-limite per il sovrano che avrebbe dovuto consistere nel lasciare libertà ai sudditi negli ambiti irrilevanti per la sicurezza dello Stato e non disciplinati dalla legge.

In ogni caso, l'archetipo hobbesiano dell'uso politico della paura a scopi di dominio era ormai consolidato.

20 L. Scuccimarra, Semantiche della paura cit., 25.

21 R. Mancini, Dialettiche della paura cit , 50.

22 R. Cornelli, La paura nel campo penale cit., p. 79.

23 L. Scuccimarra, Semantiche della paura cit., 27.

24 L. Scuccimarra, Semantiche della paura cit., 29. 
Nei regimi dispotici secondo Montesquieu proprio la paura che fa da collante tra gli individui genera anche desertificazione sociale, in quanto finisce per tenere i 'cittadini' separati tra loro ${ }^{25}$. Quale è stato il ruolo della paura nei regimi totalitari come quello fascista e nazista? Se, per natura, la paura può avere l'utilità pratica di stimolare ad evitare pericoli, questa utilità, come ha sottolineato Anna Arendt, cade in quei tipi di regime nei quali le persone sono oggetto di eliminazione non per effetto dei loro comportamenti e delle loro colpe e senza alcun riguardo «a atti o comportamenti individuali», ma soltanto perché qualificate come appartenenti a razze inferiori e ritenute inadatte a vivere ${ }^{26}$. Per non dire di quello che si suole dire anche oggi nella propaganda contro i migranti, che, non solo, mettono a rischio di 'contaminazione' o di 'corruzione' i nostri valori e i nostri principi, ma anche il nostro benessere e ci 'impoveriscono'. Dal passato al presente, dunque, in continue ricorrenze di argomenti, elaborati, innanzitutto, senza alcuna base scientifica, a soli fini di persuasione.

Una lunga digressione potrebbe riguardare ancora il fenomeno globale del terrorismo a sua volta articolato, come il lemma terrore, in categorie differenti che vanno dal terrorismo militare a quello psicologico, il cui elemento di congiunzione appare comunque consistere nel non scegliere una vittima specifica, ma nel fare delle vittime un mezzo per raggiungere un bersaglio terzo e per ottenere, per questa via, gli effetti politici desiderati con il generare il terrore di massa ${ }^{27}$.

Una prima considerazione di carattere generale, prima di passare alle declinazioni penalistiche della paura, potrebbe consistere, allora in questo. Possiamo sostenere che la paura abbia costituito nel passato, e continui a costituire nel presente, un potente strumento di creazione del consenso intorno a colui o a coloro che offrono 'una' difesa? che alimentare paure e creare nemici, per proporsi come difensori, rappresenti un meccanismo di coesione più volte utilizzato nella storia?

Quali sono le paure che pervadono la società, cosiddetta liquida, della globalizzazione? In certi casi, si tratta di paure fondate e derivanti da reazioni psico-fisiche a minacce oggettive provenienti dall'esterno ${ }^{28}$;

25 L. Scuccimarra, Semantiche della paura cit., 32.

26 L. Scuccimarra, Semantiche della paura cit., 33

27 F. Benigno, La paura estrema in politica: sui concetti di terrore e terrorismo, La paura, 55-62, 56-57.

28 L. Scuccimarra, Semantiche della paura cit., 34. 
in altri casi, invece, di paure costruite, alimentate, propagandate e rese 'pervasive' e funzionali al tentativo di affermazione di un potere che - in una sorta di circolarità tra politica e paura - si fonda sulla costruzione di figure di nemico ${ }^{29}$, le quali, a loro volta, servono alla costruzione di un'identità e di un'unità.

Si tratta allora di paure tanto più pericolose e distruttive in quanto costituiscono un'alternativa all'elaborazione di programmi e di progetti o, persino, servono come catalizzatori del consenso in assenza di una progettualità per l'azione della politica.

Sono paure, inoltre, che, secondo molti (per fortuna) potrebbero essere, invece, sfruttate per rivolgere tutte le forze verso la creazione di rapporti, di scambi, di dialogo, di legami. Si tratterebbe dunque di 'canalizzare' la paura per rivolgerla, con il concorso di 'forze più propizie' «a una società abitabile, umanizzata e democratica»"

Sappiamo, però, molto bene che i concetti di 'città' o di società, di democrazia e di giustizia non sono concetti fissi e immutabili, e esigono una continua e indefessa elaborazione e difesa da parte di tutti ${ }^{31}$. Per dirla con le parole di uno storico del diritto, si potrebbe concludere questa prima parte dicendo che la democrazia, come ogni forma di organizzazione ideata nella storia, non è una categoria astratta, ma un percorso, che esige un continuo aggiornamento per approssimazioni successive e che, quindi, il vero timore è proprio quello di smarrire il cammino.

\section{Floriana Colao}

Ringrazio anch'io il Presidente e il Consiglio dell'Istituto Lombardo per l'occasione di presentare il libro La paura. Riflessioni interdisciplinari per un dibattito contemporaneo su violenza, ordine, sicurezza e diritto di punire. Un grazie all'amica Claudia Storti e il rammarico per non essere a Milano per la pandemia, l'auspicio di prossime occasioni di incontri 'senza schermo'.

29 R. Mancini, Dialettiche della paura cit., 49, con riferimento alla cosiddetta antropologia della mancanza e della perdita.

30 R. Mancini, Dialettiche della paura cit., 48.

31 R. Cornelli, La paura nel campo penale cit., p. 88. 
Riassumo dunque alcuni temi della ricca sezione Storia del diritto penale del volume; mantengo lo stile colloquiale dell'intervento del 3 Dicembre 2020, limitandomi ad aggiungere poche note. In apertura del volume Luigi Lacchè e Massimo Meccarelli definiscono la paura «sentimento umano che si colora però diversamente a seconda dei tempi e delle latitudini $\gg^{32}$, 'storicizzando', per così dire, il sentimento sub specie iuris. L'attenzione riservata al discorso giuridico aggiunge un importante elemento alla storiografia sulla paura, a principiare dal 'classico' di Jean Delumeau - riproposto di recente - sui dispositivi e le attrezzature mentali per oggettivare l'angoscia nell'età moderna $a^{33}$. Un tema dominante nelle ricostruzioni storiche: il nesso paura-Potere, che - soprattutto nella lezione di Hobbes, 'scandalosa' all'epoca - rende il sentimento puntello della sicurezza e dunque dell'ordine, secondo la prospettiva indicata, tra gli altri, da Carlo Ginzburg ${ }^{34}$. Di «monarchia della paura» ha scritto Martha C. Nussbaum, a proposito della tendenza di taluni assetti politici ad allarmare i propri «sudditi», anche senza fondamento, con l'alterare la loro capacità di giudizio. Il volume indica la paura come un veleno per la democrazia, perché toglie alle persone libertà di pensiero e studia gli antidoti ${ }^{35}$.

Le Storie ripercorse da La paura riflettono su talune polarizzazioni, timore/paura/, panico/terrore, identità/alterità, normalità/devianza, classi lavoratrici/classi pericolose, ius puniendi pubblico/autodifesa privata. In questi schemi i contributi presentano tratti differenziali, di tempo e spazio; il saggio di Mario Piccinini differenzia il «panico» dalla «semantica della paura», rintracciandone una genealogia nel pensiero politico del primo Seicento, poi nelle più note pagine di Hobbes, Montaigne, Bacone, Shaftesbury, col panico passione politica e limite interno della

32 L. Lacchè, M. Meccarelli, Paura in criminalibus : il problema penale tra sentimento umano e dinamiche sociali, in La paura. Riflessioni interdisciplinari per un dibattito contemporaneo su violenza, ordine, sicurezza e diritto di punire, Macerata, Eum, 2019, p. 10; sul volume cfr. la densa recensione di A. Sciumè, Leggendo La paura. Riflessioni interdisciplinari per un dibattito contemporaneo su violenza, ordine, sicurezza e diritto di punire, Studi senesi, 2/2020, 427-437.

33 J. Delumeau, La paura in occidente, Storia della paura in età moderna, Milano, Il saggiatore, 2018.

34 C. Ginzburg, Paura reverenza terrore. Cinque saggi di iconografia politica, Milano, Adelphi, 2015, 57.

35 M. C. Nussbaum, La monarchia della paura. Considerazioni sulla crisi politica attuale, Bologna, Il Mulino, 2020 
politica ${ }^{36}$. La lezione dell'autore del Leviatano ritorna anche nel contributo di Emilia Musumeci sul veneficio; l'autrice mostra che l'uso del veleno - fobia ancestrale - rende il crimine sfuggente per le modalità misteriose di esecuzione. Il veneficio è 'terribile' anche per portare il marchio del tradimento, per esser propinato dai 'mani amiche', la moglie - anche nell'Ottocento sarà considerato 'delitto di donne' - i servitori, per confondersi, fin dall'etimologia, con il medicamento benefico. Emilia Musumeci ripercorre le origini romanistiche e le accezioni altomedievali del veneficio, di cui, tra l'altro, è complicato per l'interprete cogliere i tratti del tentativo. Si sofferma sulle voci dei giuristi, dal medioevo sapienziale alle trattatistica moderna, con particolare riferimento al Tractatus criminalis di Tiberio Deciani, che esamina profili sostanzali e processuali dell'atrocitas veneficii, e di Prospero Farinacci, a proposito del «crimen occultum et difficilis probationis». L'autrice coglie un avvicinamento tra veleno e pratiche magiche, opera dei giuristi e del 'senso comune'; l'aggravante dell'omicidio si colora dei tratti del maleficio delle streghe e degli untori, dalla morte nera del 1348 a quella 'manzoniana' della Milano del 1630, con gli untori - come è noto - processati e condannati. Emilia Musumeci mostra che le dottrine illuministiche e il progresso delle scienze non avrebbero scalzato certe fobie sociali; ricorda la paura che il colera del 1835 fosse stato diffuso ad arte dai medici o dai governanti, con «episodi di panico collettivo» ${ }^{37}$.

Il saggio di chi vi parla segue il pensiero giuridico sul circuito paura-potere-diritto e l'impervia costruzione di regole giuridiche 'non paurose' tra ius puniendi dello Stato e diritto dei cittadini a difendersi, tra difesa legittima ed eccesso, crime of self-defence nel modello di common law. Dall'Unità ai giorni nostri la legittima difesa è parsa legata alle percezioni sociali dei beni aggrediti o minacciati - 'la vita e la roba', il pudore e la 'fama' - e tra vittime e aggressori, i primi 'ontologicamente' onesti, separati dalla proteiforme «mala setta» nazionale. Nelle varie stagioni la legittima difesa si è rivelata banco di prova del rapporto tra Stato, crimine, beni tutelati e 'senso di giustizia' della società; la 'grande fondazione' ne cives ad arma veniant ne ha disegnato i profili di extrema ratio, in parallelo con l'uso della forza pubblica. Nella 'zona grigia' della

36 M. Piccinini, Stagioni del panico. Prime linee di ricerca, La paura, 91-112

37 E. Musumeci, La paura del crimen occultum. Declinazioni del veneficio in antico regime, La paura, 113-128, in part. 128. 
perturbatio animi la codificazione penale si è sforzata di' oggettivare la paura', tra il «timore per la sicurezza personale» del codice Zanardelli ed il «pericolo attuale» del codice Rocco. Norme generali e astratte hanno inteso scongiurare l'identificazione dell'istituto con la sua 'parente scomoda', la vendetta, la cui «fuoruscita» - come ha scritto Mario Sbriccoli - si sta rivelando «impresa non da poco». La recente riforma ispirata allo slogan 'tautologico' per cui la difesa è sempre legittima - che fa appello alla paura, frutto del 'populismo penale' - non può non rimettere all'interprete il giudizio sull' «offesa legittimata» ${ }^{38}$.

Il penale «strumento delle paure dei principi» - come scriveva Francesco Carrara - è ripercorso anche nel denso saggio di Marco Miletti, che rintraccia le interferenze tra rito giudiziario e paure, in un percorso di catarsi individuale e pacificazione pubblica. L'autore coglie che dalle origini religiose del giudizio - la giustizia in nome di Dio - si approda alle «autorità secolari» dello Stato moderno. Mostra che del processo romano canonico, comunemente definito come archetipo del 'modello inquisitorio', i tribunali laici conservarono molti tratti, la procedibilità ex officio, la delazione e la tortura per fini probatori, la compressione della difesa tecnica. Individua uno snodo nella critica illuministica di Beccaria, scegliendone un passo cruciale nel mutamento di 'senso' del processo penale, «un uomo non può chiamarsi reo prima della sentenza del giudice». Nel saggio di Miletti Montesquieu è richiamato per evocare il rispetto della forme processuali, pena gli animi «in preda alla paura», $i$ «volti pallidi». Per l'Illuminismo il processo penale non doveva infatti atterrire; si stagliava - in una pagina di Filangieri lo «spavento del malvagio», da combinare però con la «sicurezza dell'innocente». Miletti mostra poi che nell'Italia liberale la penalistica tematizzava il rito o come «incubo» dei «galantuomini» - memorabili le pagine di Carrara sulla «immoralità del carcere preventivo», che domoralizzava anche l'opinione pubblica - o come «difesa sociale contro i delinquenti». Nel primo caso rilevava la paura dell'inquisito, nel secondo quella della società; l'autoritarismo fascista avrebbe accentuato quest'ultimo profilo, a scapito delle garanzie. Neppure il 'modello accusatorio', seppure 'all'italiana' - delineato nell'unico codice della Repubblica - avrebbe scalzato questo tratto permanente della giustizia

38 F. Colao, Paura e legittima difesa. Questioni di «moderame» tra Otto e Novecento, La paura, 129-146, in part. 145. 
penale. In età repubblicana la paura dell'avviso di garanzia - 'sostitutivo' del castigo - pare oltretutto complicata dal conflitto tra potere politico e magistratura e dalla mediatizzazione della giustizia penale, esemplare la vicenda della prescrizione e del 'fine processo mai'; da qui una più che fondata «ipocondria giudiziaria» ${ }^{39}$.

Dal penale la paura sembra irrompere anche nel campo della codificazione civile, come mostra il saggio di Giacomo Pace Gravina. L'autore osserva che nel codice civile non ci aspetteremmo una nozione precisa della paura, elemento essenziale dei reati - l'estorsione, le minacce, il recente stalking - e base della legittima difesa. Il saggio ne dimostra la notevole rilevanza nel diritto delle obbligazioni e nel diritto matrimoniale e successorio, con l'esposizione di sè e i beni «ad un male ingiusto e notevole». La paura rileva come specchio della violenza, che costituisce uno dei vizi del consenso nel contratto (artt. 1434 e seguenti del codice civile del 1942). Pace ricostruisce una «genealogia del timore» della scienza civilistica, pur rimasta estranea alle ricerche dei fisiologi, psicologi, psichiatri, un nome per tutti Angelo Mosso, La Paura, Milano 1884. Dal lavoro dei giuristi sulle fonti romanistiche e canonistiche allo sguardo dell'Encyclopedie Pace coglie la differenza tra una paura grave - quella della morte - che colpisce anche uomini forti; una leggera, atta a turbare uomini pavidi o timidi. Il legislatore napoleonico pare mutuare dall'Illuminismo un modello razionalistico, col sostituire l'bomo constantissimo con quello 'ragionevole'; nel paradigma della razionalità la paura per essere rilevante doveva poter incidere sulla volontà della maggior parte degli individui, con un riferimento alla 'media dei soggetti'. Pace osserva che il codice civile italiano del 1865 parla di «persona sensata», con un 'nocciolo duro' di inevitabile relativismo nel considerare il turbamento dell'animo - un terrore che paralizza - in grado di viziare il consenso, rimesso al prisma della giurisprudenza in tema di annullamento di obbligazioni e contratti conclusi per violenza morale ${ }^{40}$.

La dialettica paura/potere, tra storia e sistema, è particolarmente

39 M.N. Miletti, La paura del processo. Spunti nella penalistica italiana (secoli XVIII-XX), La paura, 199-238, in part. 236.

40 G. Pace Gravina, «I contratti conclusi sotto l'impero tirannico della paura». L'accezione del timore nel diritto delle obbligazioni dell'età dei Codici, La paura, 147. 158 , in part. 157 
evidente nel saggio di Luigi Lacchè; alla ricerca di «archetipi e radici» il promotore dei Quaderni di storia del penale e della giustizia afferma che «l'ordine giuridico liberale riflette un'idea di sovranità e uno stile di pensiero che hanno a che fare con la razionalità del governo di soggetti dotati di autonomia morale e piena capacità decisionale e che esclude, in tutto o in parte, la non-soggettività o soggettività altre da sottoporre a dispositivi disciplinari». Nel delineare la «genealogia delle classi pericolose» Lacchè argomenta che il ceto politico postunitario fu incapace di rispondere alla richiesta di partecipazione di soggetti percepiti come elemento di disturbo della 'Libertà proprietaria' dell'bomo oeconomicus. Da qui la distinzione anche antropologica classi laboriose/classi pericolose, l'avvicinamento del povero - già alter Christus - al delinquente, lo straccione, il vagabondo, che minaccia l'ordine della città, esemplari le pagine da Victor Hugo a Dickens. Quanto alla politica, Lacchè coglie in particolare nel discorso di Thiers del 1850 il paradigma della separazione tra popolo laborioso e feccia, branco che spaventa Parigi e la Francia. Analizza poi il contributo centrale offerto dall'antropologia criminale alla definizione dell'uomo pericoloso, oltre che delinquente; Cesare Lombroso ed adepti sembrano rendere di senso comune l'alterità tra normali e poveri, criminali, folli, nel segno di una inferiorità biologica e sociale, che necessita di misure di prevenzione praeter delictum, punto di crisi del principio di legalità. Il denso saggio si sviluppa tra prospettiva storica e la continuità della messa in scena di un 'sempreverde' confine tra il noi ed il 'nemico di turno', il «capro espiatorio»; la paura dei migranti - come nell'Ottocento 'minaccia del numero' - pare suggerire un «ritorno al futuro», la «costituzione materiale del sistema repressivo e preventivo che consenta regimi diversi e attenuati di legalità»; questa, per Lacchè, la «vera posta il gioco» ${ }^{41}$.

«La storica paura dell'immigrazione di massa» è ricostruita, anche con una efficace iconografia, dal saggio di Michele Pifferi. L'autore premette che oggi per la società non rileva il timore della libertà di migrare dell'individuo - già tematizzata da Ugo Grozio - ma la dimensione collettiva del fenomeno, la discessio singulorum, con il rischio e la paura per cui «civilis societas subsistere non possit». In un ampia prospettiva comparata Pifferi ricorda che l'articolo 3 del codice civile del Regno

41 L. Lacchè, La paura delle «classi pericolose». Ritorno al futuro ?, La paura, $159-178$, in part. 178. 
d'Italia considerava il diritto di soggiorno dello straniero un diritto naturale e civile. Osserva che nel 1865 erano gli Italiani ad emigrare, a confronto di una scarsa immigrazione; e che, interpretando l'art. 3, la dottrina poneva comunque il tema dell'eccezione al principio del 'riconoscimento del diritto' in caso di «invasione di stranieri». Quanto agli Stati uniti - 'laboratorio' del tema - Pifferi mostra che le politiche restrizioniste, dopo una lunga fase di open doors, erano sorrette dal consenso dei Sindacati, allarmati dalla concorrenza salariale e il benessere a rischio di fronte alla «Alien invasion». Il saggio illustra poi che nel 1882 il governo inaugurava un regime giuridico discriminatorio nei confronti degli asiatici; la rivista Wasp raffigurava il cinese fornito di undici mani, per svolgere altrettanti mestieri, sottratti ai nativi. Nei popolari cartoons erano i lavoratori - un francese, un ebreo, un afroamericano, un irlandese, un veterano della guerra civile - ad erigere un muro contro l'arrivo del pericolo giallo, che abbatte la sua muraglia, ostacolo alla partenza. Pifferi ricorda inoltre l'Alien Act del Parlamento inglese, 1905, con la categoria, vaga, degli indesiderabili, e con l'argomento del pericolo per il welfare state, perimetrato sui cittadini entro i confini nazionali. Mostra la potenza comunicativa delle «costanti retoriche della paura», il «suicidio della razza e criminalità degli immigrati»; ma anche la forza dell'argomento dell'apprendimento dei valori della democrazia americana, condizione di diventare buoni cittadini. La conclusione è un problema aperto: le leggi sul controllo dei confini hanno bisogno della paura per legittimare il loro carattere extra ordinem; il diritto in materia deve garantire la sicurezza, ma può non essere in grado di rispondere alle istanze securitarie, propagandate ma anche reali o percepite tali ${ }^{42}$.

Nel dramma di questi giorni - di questi tanti mesi - è la pandemia ad essersi imposta come paura dominante, di morire e soprattutto di veder morire i propri cari; anche se Danilo Zolo anni addietro aveva ricordato la «paura della morte [...] pane quotidiano di una umanità che vive nella disperazione» ${ }^{43}$. Quanto al 'governo' della paura, alla relazione tra paura e potere, mi sembrano importanti le considerazioni del saggio di Claudia Storti, posto a conclusione del volume. «La democra-

42 M. Pifferi, Paure dello straniero e controllo dei confini. Una prospettiva storico-giuridica, La paura, 179-198.

43 D. Zolo, Sulla paura. Fragilità, aggressività, potere, Milano, Feltrinelli, 2011. 
zia, nella quale tutti noi continuiamo a credere» - scrive l'autrice - è «paradigma di tutela dei diritti individuali e di ordine contro la forza e la violenza $[\ldots .$.$] tuttavia la crisi di funzionamento del modello democra-$ tico è sotto gli occhi di tutti» ${ }^{44}$. Tra storia e drammatico presente chiudo con tanti dubbi ed una considerazione: se non vogliamo rassegnarsi alla prospettiva per cui l'ordine liberal-democratico è inadatto a risolvere i problemi della società - anche nel vuoto di intelligenza politica - in risposta alle legittime paure per tutti si pone il tema culturale della ricerca di strumenti soprattutto giuridici all'altezza dei problemi, in grado di incanalare le soluzioni nell'alveo e nel rispetto dei principi costituzionali, sovranazionali ed internazionali.

44 C. Storti, A proposito di uso politico della paura, La paura, 295-299, in part. 298. 
